\title{
Optimal Denoising in Redundant Bases
}

\author{
Martin Raphan, Member, IEEE, Eero P. Simoncelli, Senior Member, IEEE
}

\begin{abstract}
Image denoising methods are often designed to minimize mean squared error (MSE) within the subbands of a multi-scale decomposition. But most high quality denoising results have been obtained with overcomplete representations, for which minimization of MSE in the subband domain does not guarantee optimal MSE performance in the image domain. We prove that despite this suboptimality, the expected imagedomain MSE resulting from applying estimators to subbands that are made redundant through spatial replication of basis functions (e.g., cycle spinning) is always less than or equal to that resulting from applying the same estimators to the original non-redundant representation. In addition, we show that it is possible to further exploit overcompleteness by jointly optimizing the subband estimators for image-domain MSE. We develop an extended version of Stein's unbiased risk estimate (SURE) that allows us to perform this optimization adaptively, for each observed noisy image. We demonstrate this methodology using a new class of estimator formed from linear combinations of localized "bump" functions that are applied either pointwise or on local neighborhoods of subband coefficients. We show through simulations that the performance of these estimators applied to overcomplete subbands and optimized for image-domain MSE is substantially better than that obtained when they are optimized within each subband. This performance is, in turn, substantially better than that obtained when they are optimized for use on a non-redundant representation.
\end{abstract}

IEEE EDICS: RST-DNOI

\section{INTRODUCTION}

Image denoising has undergone dramatic improvement over the past decade, due to both the development of linear decompositions that simplify the characteristics of the signal, and to new estimators that are optimized for those characteristics. A standard methodology proceeds by linearly transforming the image, operating on the transform coefficients with nonlinear estimation functions, and then inverting the linear transform to obtain the denoised image. Estimation functions generally take the form of "shrinkage" operators that are applied independently to each transform coefficient (e.g., [1], [2], [3], [4], [5], [6], [7], [8]), or are applied to neighborhoods of coefficients at adjacent spatial positions and/or from other subbands (e.g., [9], [10], [11], [12]).

The choice of estimation function is an essential part of the denoising problem. From a statistical perspective, one may use a prior probability model for the transform coefficients (either assumed, or fit to a set of clean training images), and then use this to derive a Bayes-optimal estimator. Alternatively, one may directly assume a parametric form for the estimation function, and select parameters by optimizing performance on a training set containing pairs of clean images and their noisy

M. Raphan and E. P. Simoncelli are with the Howard Hughes Medical Institute, Center for Neural Science, and the Courant Institute of Mathematical Sciences, New York University, New York, NY, 10003.

E-mail: raphan@cims.nyu.edu, eero.simoncelli@nyu.edu counterparts [e.g., 13]. As described, these methodologies require explicit information about clean images, either through samples or knowledge of the prior distribution, both of which will typically reflect the statistics of heterogeneous ensembles of images. However, there are ways of adaptively optimizing the parameters of the density model for the particular image being denoised. For example, an "empirical Bayes" estimator may be derived from a prior density whose parameters are chosen to best account for the observed noisy image (typically, by maximizing likelihood) [3]. A parametric estimator may also be adaptively optimized by minimizing Stein's unbiased estimate of risk (SURE) [14], which provides an approximation of the mean squared error (MSE) as a function of the observed noisy data. [2], [15] Assuming there is enough data to capture the statistics of a given image, adaptive methods will always perform better than those optimized for a heterogeneous ensemble of images.

Although it is less well understood, the choice of linear transform also has an impact on the quality of denoising results. Multi-scale decompositions are a typical choice, and both empirical Bayes methods [3], [5], [8], and SURE adaptive methods have been used to optimize scalar [2], [16], [17], [18] and joint [15] estimators for application to subbands of multi-scale decompositions. Empirical evidence indicates that redundant (overcomplete) multi-scale representations are more effective than orthonormal representations [19], [20]. This fact is somewhat mysterious, since the estimators are generally optimized for MSE within individual subbands, which (for a redundant basis) is not the same as the MSE in the image domain. Recent work provides an interesting explanation for this phenomenon by interpreting shrinkage in overcomplete representations as the first iteration of a Basis Pursuit denoising algorithm [21].

In this paper, we prove that application of denoising functions to subbands made overcomplete through cycle spinning or elimination of decimation is guaranteed to be no worse in MSE (and is in practice significantly better) than applying the same functions in an orthonormal basis. This method of denoising, however, does not take full advantage of the redundancy, and further improvements may be obtained by jointly choosing subband denoising functions that optimize MSE in the image domain. We provide a method for this imagedomain optimization by extending SURE to approximate the image-domain MSE that results from applying estimation functions to coefficients of a redundant basis. We develop a family of parametric estimators based on a superposition of localized "bump" functions, and demonstrate through simulations that optimizing the image-domain SURE of these estimators applied within undecimated wavelet subbands leads to significant performance improvements over optimizing the subband-domain SURE of the same estimators. A preliminary 
version of this work has been presented in [22].

\section{Redundancy Improves Performance}

Given a noisy image $Y$, we wish to compute an estimate of the original (clean) image $\hat{X}(Y)=f(Y)$, where the estimator $f$ is selected from a family $\mathcal{F}$ to minimize the MSE:

$$
f_{\text {opt }}=\arg \min _{f \in \mathcal{F}} E\left\{|X-f(Y)|^{2}\right\},
$$

and $E\{\}$ indicates the expected value. We may consider $X$ to be fixed but unknown (the so-called "frequentist" perspective), with the expectation taken over $Y$ conditioned on $X$. Alternatively, we may consider $X$ to be a sample drawn from some prior probability distribution $p_{x}(X)$ (the "Bayesian" perspective). In this case, the expectation is taken jointly over $X$ and $Y$, or (equivalently) over $Y$ conditioned on $X$, and then over $X$.

A common practice in image denoising is to use estimators that act on a linearly transformed version of the corrupted image, $U=W Y$. Here, $W$ can be a complete or overcomplete linear transformation (an $m$ by $n$ matrix, $m \geq n$, where $n$ is the dimension of image space), that has a left inverse $W^{\dagger}$ such that $W^{\dagger} W=I d$. In this section, we assume that the transform is a tight frame, for which $W^{\dagger}=W^{T}$. This includes orthogonal, cycle-spun and undecimated wavelet transforms, as well as other overcomplete decompositions such as the steerable pyramid [23], curvelets [24], or complex dual-tree wavelets [25].

In this situation, the estimate is computed by transforming the original signal, applying an estimator $f_{u}$ in the transform domain, and then inverse transforming with $W^{T}$ :

$$
f(Y)=W^{T} f_{u}(W Y),
$$

The MSE incurred in using this estimator is thus:

$$
E\left\{\left|X-W^{T} f_{u}(W Y)\right|^{2}\right\}=E\left\{\left|W^{T}\left(V-f_{u}(U)\right)\right|^{2}\right\}
$$

where $V=W X$, the transform of the clean image. Note that in the case when $W$ is orthogonal, the transform preserves vector lengths (as does $W^{T}$ ), and the MSE reduces to $E\left\{\left|\left(V^{o}-f_{u}\left(U^{o}\right)\right)\right|^{2}\right\}$, where the superscripts on $U, V$ are a reminder that the coefficients were obtained from an orthogonal transform. In the next sections, we explain why and under what conditions the performance of a denoising function on an orthonormal wavelet basis can be improved by adding redundancy to the transform through cycle spinning or elimination of decimation. For didactic purposes, we will consider cycle spinning.

\section{A. Scalar estimators}

Consider an estimator $f_{u}$ composed of scalar functions $f_{i}$ that operate pointwise on the transform coefficients. Typically, the transform coefficients are partitioned into subbands $\left\{\mathcal{S}_{k} ; k=1,2, \ldots K\right\}$, corresponding to shifted versions of the same basis function, all of which can be assumed to have the same marginal statistical properties. In this case, the same estimator will be applied to all coefficients within each subband, and the MSE can be partitioned into a sum of MSEs on each subband. If we assume an orthogonal transform, we can write the MSE as:

$$
\begin{aligned}
E\{\mid & \left.\left.\left(V^{o}-f_{u}\left(U^{o}\right)\right)\right|^{2}\right\} \\
& =E\left\{\sum_{k} \sum_{i \in \mathcal{S}_{k}}\left|V_{i}^{o}-f_{k}\left(U_{i}^{o}\right)\right|^{2}\right\} \\
& =\sum_{k} \sum_{i \in \mathcal{S}_{k}} E\left\{\left|V_{i}^{o}-f_{k}\left(U_{i}^{o}\right)\right|^{2}\right\}
\end{aligned}
$$

Now consider a cycle-spun decomposition, in which the basis functions of the original orthonormal representation are replicated at $N$ translated positions [19]. Each subband will contain $N$ times as many coefficients as the corresponding subband of the orthonormal representation. In order to form a tight frame, each basis function must be divided by a factor of $\sqrt{N}$, relative to those of the orthonormal representation. Thus, if $f_{k}$ is the marginal function used to denoise coefficients in the orthonormal wavelet representation, the corresponding function that should be applied in the cycle-spun decomposition is

$$
f_{k}^{c}(u)=\frac{1}{\sqrt{N}} f_{k}(\sqrt{N} u)
$$

In addition to the tight frame assumptions, we also assume that relationship between the noisy and clean coefficients in each band will be the same (up to a factor of $\sqrt{N}$ ) in the cycle-spun representation as in the orthonormal representation. Specifically, we assume the joint statistics of the rescaled cycle-spun coefficients, $\left(\sqrt{N} U^{c}, \sqrt{N} V^{c}\right)$ are the same as those of the orthogonal coefficients, $(U, V)$. This allows us to rewrite Eq. (2), in terms of the cycle-spun coefficients:

$$
\begin{aligned}
\sum_{k} & \sum_{i \in \mathcal{S}_{k}} E\left\{\left|V_{i}^{o}-f_{k}\left(U_{i}^{o}\right)\right|^{2}\right\} \\
& =\sum_{k} \frac{1}{N} \sum_{i \in \mathcal{S}_{k}} E\left\{\left|\sqrt{N} V_{i}^{c}-f_{k}\left(\sqrt{N} U_{i}^{c}\right)\right|^{2}\right\} \\
& =\sum_{k} \sum_{i \in \mathcal{S}_{k}} E\left\{\left|V_{i}^{c}-\frac{1}{\sqrt{N}} f_{k}\left(\sqrt{N} U_{i}^{c}\right)\right|^{2}\right\} \\
& =\sum_{k} \sum_{i \in \mathcal{S}_{k}} E\left\{\left|V_{i}^{c}-f_{k}^{c}\left(U_{i}^{c}\right)\right|^{2}\right\} \\
& =E\left\{\left|V^{c}-f_{u}^{c}\left(U^{c}\right)\right|^{2}\right\}
\end{aligned}
$$

Combining Eq. (2) and Eq. (3), we see that the MSE in the orthonormal case is equal to the total subband-domain MSE in the cycle-spun case.

Now it is straightforward to compare the MSE in the orthonormal case, as given by Eqs. (2) and (3), to the imagedomain MSE incurred with cycle-spun denoising, as specified by Eq. (1):

$$
\begin{aligned}
E\left\{\left|V^{o}-f_{u}\left(U^{o}\right)\right|^{2}\right\} & =E\left\{\left|V^{c}-f_{u}^{c}\left(U^{c}\right)\right|^{2}\right\} \\
& \geq E\left\{\left|W^{T}\left(V^{c}-f_{u}^{c}(U)\right)\right|^{2}\right\}
\end{aligned}
$$

where the inequality holds because $W$ is an overcomplete tight frame, and thus $W^{T}$ is a projection operator. That is, the image-domain MSE for the cycle-spun case is always less than or equal to that for the cycle-spun case. Note that we have assumed nothing regarding the form of the estimators 
$f_{k}$. We also assumed very little about the corruption process - only that it is stationary. The result may be easily extended to undecimated wavelets, in which the number of coefficients in each band will be multiplied by a different factor.

The upshot is that, as long as the joint statistics of pairs of clean and noisy coefficients don't change when going to a redundant basis (as is the case for stationary image and noise statistics) then the performance of any marginal denoising function on the redundant basis is guaranteed to be no worse then the corresponding operations performed on the orthonormal representation. In particular, if we choose an optimal estimator to denoise each subband of an orthogonal transform, the optimal estimator for the redundant basis will be the same (up to rescaling by $\sqrt{N}$ ), and the performance of the redundant system will generally be better than that of the orthogonal one.

\section{B. Context estimators}

Thus far we have been discussing scalar estimation functions. However, recent literature has demonstrated that substantial improvements can be achieved with estimators that operate on the surrounding "context" of multi-scale coefficients (e.g., [9], [10], [26], [11], [12], [15], [27], [28]). In general, the surrounding neighborhood can include coefficients within the same subband, as well as coefficients in other subbands, and the neighborhoods are generally overlapping (i.e., each coefficient belongs to more than one neighborhood). For our purposes, we handle the overlap of neighborhoods by computing an estimate only for the center coefficient (as in [10], [11], [12]):

$$
\hat{V}_{i}\left(\mathbf{U}_{i}\right)=f_{k}\left(\mathbf{U}_{i}\right) .
$$

Thus, each coefficient is denoised as a function of its surrounding neighbors.

Analogous to the scalar case, we can prove that, for certain neighborhood types, applying a vector denoising function to the subbands of a representation made redundant by spatial replication of orthonormal basis functions leads to improvement in denoising performance over using the original orthonormal basis. In an orthonormal basis, Eq. (2) for the MSE is replaced by

$$
E\left\{\left|\left(V^{o}-f_{u}\left(U^{o}\right)\right)\right|^{2}\right\}=\sum_{k} \sum_{i \in \mathcal{S}_{k}} E\left\{\left|V_{i}^{o}-f_{k}\left(\mathbf{U}_{i}^{o}\right)\right|^{2}\right\} .
$$

We now want to express this error in terms of the neighborhoods of the redundant representation. In order to do so, we must ensure that the neighborhoods in both representations have the same statistics (appropriately scaled). The easiest way to achieve this is to use the same sampling pattern. In a cyclespun representation we simply draw adjacent neighbors from within the shifted copies of the original orthonormal decomposition. In an undecimated wavelet, we must choose neighbors of a coefficient on a subsampled lattice, with subsampling factor the same as that used to form the associated orthogonal wavelet subband. Thus, as in the scalar case, we may express the MSE for the the orthogonal wavelets in terms of the cyclespun wavelets:

$$
\begin{gathered}
E\left\{\sum_{k} \frac{1}{N} \sum_{i \in \mathcal{S}_{k}}\left|\sqrt{N} V_{i}^{c}-f_{k}\left(\sqrt{N} \mathbf{U}_{i}^{c}\right)\right|^{2}\right\} \\
=E\left\{\sum_{k} \sum_{i \in \mathcal{S}_{k}}\left|V_{i}^{c}-f_{k}^{c}\left(\mathbf{U}_{i}^{c}\right)\right|^{2}\right\}
\end{gathered}
$$

where, analogous to the marginal case,

$$
f_{k}^{c}(\mathbf{U})=\frac{1}{\sqrt{N}} f_{k}(\sqrt{N} \mathbf{U}) .
$$

The rest of the proof, and the extension to undecimated wavelets is completed as in the marginal case. ${ }^{1}$

\section{STEIN'S LEMMA AND OPTIMAL DENOISING}

The proofs of the previous section suggest that we can improve the performance of subband estimators that are optimized separately for each subband by increasing the redundancy of the subbands. But this does not tell us how much gain we can expect. Nor does it imply that increasing redundancy alone will allow the overall system to obtain the best possible performance, as measured by MSE in the image domain. In particular, it is apparent that jointly optimizing the estimators that are applied to each subband will always lead to performance that is as good as, or better than, that obtained with estimators that are independently optimized. As such, the remainder of this article examines the gains that are attainable through the two performance-enhancing techniques of increasing redundancy, and joint optimization.

In order to examine performance gains, we need to select a means of optimizing the estimators. Reconsidering Eq. (1), it would seem that choosing an optimal estimator requires that we know either the clean image, $X$ (the frequentist view), or the statistics of the clean image (the Bayesian view). But in 1981, Charles Stein derived an alternative expression for the MSE, for the special case when $Y$ is derived from $X$ by addition of independent zero-mean white Gaussian noise with known variance $\sigma^{2}$ [14]. Recasting the estimator in a form that is relative to the identity, $\hat{X}(Y)=Y+g(Y)$, Stein's expression may be written as:

$$
\begin{aligned}
& E\left\{|X-(Y+g(Y))|^{2}\right\}= \\
& \quad E\left\{|g(Y)|^{2}+2 \sigma^{2}(\nabla \cdot g)(Y)\right\}+\text { const. }
\end{aligned}
$$

where the constant does not depend on the estimator function $g$. Thus, by assuming knowledge of the statistical relationship between $X$ and $Y$, the MSE may be expressed without explicit reference to either $X$ or $p_{x}(X)$. We have recently shown that this concept may be generalized to several types of nonGaussian noise, as well as a variety of non-additive corruption processes [29].

The expression in the curly braces of Eq. (5), known (up to an additive constant) as Stein's unbiased risk estimate (SURE),

\footnotetext{
${ }^{1}$ Note that it is sufficient for the transform domain MSE of the overcomplete denoiser to be no greater than that for the orthogonal wavelet denoiser. Therefore, if we use a different sampling lattice, the proof will still hold, as long as the neighborhoods of the coefficient in the redundant basis can do at least as good a job of estimating that coefficient.
} 
may be evaluated on a single observation $Y$ to produce an unbiased estimate of the MSE. As a consequence, the optimal estimation function $g_{\mathrm{opt}}$ can be approximated by minimizing the SURE expression:

$$
g_{\text {opt }} \approx \arg \min _{g \in \mathcal{G}}\left\{|g(Y)|^{2}+2 \sigma^{2}(\nabla \cdot g)(Y)\right\}
$$

Although the derivation of this expression is relatively simple, it leads us to the somewhat counterintuitive conclusion that the estimator $g$ may be optimized without explicit knowledge of the clean signal $X$, either in the form of training data or a probability model.

Further intuition regarding the optimal choice of estimator can be gained by considering the case when $g$ is a scalar operator, for which the SURE solution reduces to:

$$
g_{\text {opt }} \approx \arg \min _{g \in \mathcal{G}}\left\{\sum_{i} g\left(Y_{i}\right)^{2}+2 \sigma^{2} \sum_{i} g^{\prime}\left(Y_{i}\right)\right\} .
$$

where the $Y_{i}$ are simply the components of vector $Y$. First, note that the summations can now be viewed as sample average approximations of the expectation in Eq. (5). With this interpretation, we see that the optimal function $g$ depends only on the marginal density of the components of $Y$, regardless of any dependencies between them. The first term of the objective function seeks to select a $g$ that has small amplitude at locations of the data points $Y_{i}$, and the second term seeks a $g$ with large negative derivative at the data points. Together this means that the optimal denoiser $\hat{x}(y)=y+g(y)$ should "shrink" the observations toward those locations where the data are most concentrated.

SURE was first applied to image denoising by Donoho, who used it to determine optimal threshold values for softthreshold shrinkage functions applied to orthogonal wavelet subbands [2]. It has also been used in conjunction with cyclespun wavelets [19], alternative pointwise nonlinear estimators [16], two-component Gaussian mixture models [17], and for an inter-scale contextual estimator [15], and most recently, to optimize a 2-parameter scalar subband estimator in the image domain [18].

\section{A. SURE for correlated Gaussian noise}

SURE was developed primarily for the case of uncorrelated Gaussian noise. But since we wish to use it to optimize estimators that will be applied to subbands of redundant transforms, we will need to consider conditions where the noise has taken on transform-induced correlations. We can derive an extension of Stein's expression for MSE in the case of correlated noise. Specifically, when $Y$ is derived from $X$ by addition of independent Gaussian noise with covariance $C_{n}$ :

$$
\begin{aligned}
& E\left\{|X-(Y+g(Y))|^{2}\right\}= \\
& E\left\{|g(Y)|^{2}+2 \cdot \operatorname{tr}\left(C_{n} \frac{\partial g}{\partial y}(Y)\right)\right\}+\text { const. }
\end{aligned}
$$

where $\operatorname{tr}(\cdot)$ indicates the trace of a matrix, $\frac{\partial g}{\partial y}$ is the Jacobian matrix, and the constant does not depend on the choice of estimator.
To derive this result, we first expand the left side of Eq. (7):

$$
\begin{aligned}
& E\left\{|X-(Y+g(Y))|^{2}\right\}= \\
& \quad E\left\{|g(Y)|^{2}+2(Y-X) \cdot g(Y)\right\}+\text { const. }
\end{aligned}
$$

Since the first term matches that of Eq. (7), we need only show that

$$
E\{(Y-X) \cdot g(Y)\}=E\left\{\operatorname{tr}\left(C_{n} \frac{\partial g}{\partial y}(Y)\right)\right\}
$$

The result may be proved by explicitly writing the integral expression for the expectation over $\mathrm{Y}$ conditioned on $\mathrm{X}$, and then integrating by parts:

$$
\begin{aligned}
& E\left\{\operatorname{tr}\left(C_{n} \frac{\partial g}{\partial y}(Y)\right)\right\} \\
& =\frac{1}{Z} \sum_{i j} \int e^{-\frac{1}{2}(\mathbf{y}-\mathbf{x})^{T} C_{n}^{-1}(\mathbf{y}-\mathbf{x})}\left(C_{n}\right)_{i j} \frac{\partial g_{i}}{\partial y_{j}}(\mathbf{y}) d \mathbf{y} \\
& =\frac{-1}{Z} \sum_{i j} \int g_{i}(\mathbf{y})\left(C_{n}\right)_{i j} \frac{\partial}{\partial y_{j}} e^{-\frac{1}{2}(\mathbf{y}-\mathbf{x})^{T} C_{n}^{-1}(\mathbf{y}-\mathbf{x})} d \mathbf{y} \\
& =\frac{1}{Z} \sum_{i j} \int g_{i}(\mathbf{y})\left(C_{n}\right)_{i j}\left(C_{n}^{-1}(\mathbf{y}-\mathbf{x})\right)_{j} e^{-\frac{1}{2}(\mathbf{y}-\mathbf{x})^{T} C_{n}^{-1}(\mathbf{y}-\mathbf{x})} d \mathbf{y} \\
& =\frac{1}{Z} \sum_{i} \int g_{i}(\mathbf{y})\left(y_{i}-x_{i}\right) e^{-\frac{1}{2}(\mathbf{y}-\mathbf{x})^{T} C_{n}^{-1}(\mathbf{y}-\mathbf{x})} d \mathbf{y} \\
& =E\{(Y-X) \cdot g(Y)\}
\end{aligned}
$$

where $Z=\left|2 \pi C_{n}\right|^{\frac{1}{2}}$. Note that we can obtain the same result when $\mathrm{X}$ and $\mathrm{Y}$ are both random variables (the Bayesian case) by taking the expectation over $\mathrm{X}$.

\section{B. SURE for transform-domain estimators}

Stein's expression may also be easily extended to estimators that operate on a linearly transformed version of the signal. Analogous to the development in Section II, suppose we have a family of estimators $\left\{u+g_{u}(u): g_{u} \in \mathcal{G}_{U}\right\}$ that are applied to a transformed version of the noisy signal, $U=W Y$. The estimate is computed by transforming with $W$, applying $g_{u}$, and then inverse transforming with $W^{\dagger}$ :

$$
\begin{aligned}
\hat{X}(Y) & =W^{\dagger}\left(W Y+g_{u}(W Y)\right) \\
& =Y+W^{\dagger} g_{u}(W Y) .
\end{aligned}
$$

Given the form of this estimator, $g(Y)$ will be given by $W^{\dagger} g_{u}(W Y)$ so that

$$
\frac{\partial g}{\partial y}(Y)=W^{\dagger} \frac{\partial g_{u}}{\partial u}(U) W
$$

Inserting this into in Eq. (7) gives following expression for the image-domain MSE:

$$
\begin{aligned}
E & \left\{|X-(Y+g(Y))|^{2}\right\} \\
& =E\left\{\left|W^{\dagger} g_{u}(U)\right|^{2}+2 \cdot \operatorname{tr}\left(C_{n} W^{\dagger} \frac{\partial g_{u}}{\partial u}(U) W\right)\right\} \\
& =E\left\{\left|W^{\dagger} g_{u}(U)\right|^{2}+2 \cdot \operatorname{tr}\left(W C_{n} W^{\dagger} \frac{\partial g_{u}}{\partial u}(U)\right)\right\}
\end{aligned}
$$

As before, the expression in braces is an unbiased estimate of the MSE and can be optimized even over a single sample 
of $U$. For simplicity, in what follows we will again assume that the transform is a tight frame $\left(W^{\dagger}=W^{T}\right)$. Notice that in this case, the second term in Eq. (13) contains the covariance of the noise in the transform domain, $W C_{n} W^{T}$. In what follows we will assume that the additive noise is white (i.e., $C_{n}$ is a multiple of the identity matrix), but most of the results also hold for correlated noise. The $W^{T}$ in the first term of Eq. (13) projects the estimated values back into the image domain before computing the norm. For an overcomplete representation, this term allows us to choose the optimal denoiser in the transform domain that minimizes MSE in the image domain.

\section{EXAMPLE: SCALAR ESTIMATORS IN OVERCOMPLETE BASES}

Suppose now that $g_{u}$ consists of scalar functions $g_{i}$ that operate pointwise on (i.e., on each element of) $U$. The unbiased risk estimator in Eq. (13) becomes

$$
\left|W^{T} g_{u}(U)\right|^{2}+2 \sigma^{2} \sum_{i} n_{i i} g_{i}^{\prime}\left(U_{i}\right)
$$

where $n_{i j}$ is the $i j^{t h}$ element of $W W^{T}$ (the dot product of the $i^{t h}$ and $j^{t h}$ rows of $W$ ). If the transform coefficients are partitioned into subbands, as described in Section II, with the same estimator applied to all coefficients within a subband, the unbiased risk estimator becomes

$$
\left|W^{T} g(U)\right|^{2}+2 \sigma^{2} \sum_{k} n_{k} \sum_{i \in \mathcal{S}_{k}} g_{k}^{\prime}\left(U_{i}\right)
$$

where $n_{k}$ is the common value of $n_{i i}$ for $i \in \mathcal{S}_{k}$. For a single transformed image $U=W Y$, this expression provides a criterion for choosing $\left\{g_{k}\right\}_{k=1}^{K}$ so as to minimize the imagedomain MSE. ${ }^{2}$

We will compare this with the denoiser resulting from minimizing the SURE expression of Eq. (7) independently for each subband. Specifically, assuming again that the $\left\{g_{k}\right\}$ are marginal functions, and given that the marginal variance of the Gaussian noise in the $k^{t h}$ subband is $n_{k} \sigma^{2}$, the SURE approximation of the MSE in the $k^{t h}$ subband is

$$
\sum_{i \in \mathcal{S}_{k}} g_{k}\left(U_{i}\right)^{2}+2 \sigma^{2} n_{k} \sum_{i \in \mathcal{S}_{k}} g_{k}^{\prime}\left(U_{i}\right)
$$

Since the SURE expressions for each of the functions $\left\{g_{k}\right\}$ depend only on the coefficients of their associated subband, $\mathcal{S}_{k}$, we may combine them into a single objective function for the transform-domain MSE:

$$
\sum_{k} \sum_{i \in \mathcal{S}_{k}} g_{k}\left(U_{i}\right)^{2}+2 \sigma^{2} \sum_{k} n_{k} \sum_{i \in \mathcal{S}_{k}} g_{k}^{\prime}\left(U_{i}\right) .
$$

We can see that this expression for the transform-domain SURE differs from the image-domain SURE of Eq. (14) only in the $L_{2}$-norm expressed by the first term: image-domain SURE is computed on the values of $g_{k}\left(U_{i}\right)$ after projecting back into the image domain, thus explicitly taking into account the interactions that occur when the denoised coefficients are recombined in the image domain. We can, therefore, hope to

\footnotetext{
${ }^{2}$ This result for scalar denoisers and white noise was derived in [22], [18].
}

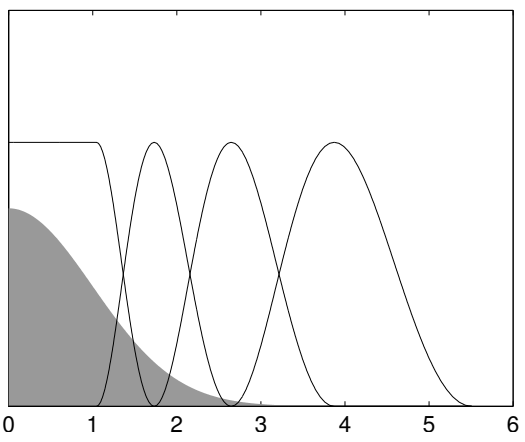

Fig. 1. Linear basis of "bump" functions, $b_{k}$, used to form $g_{\theta}(y)$. Positions of these functions are adapted to the noise level: Abscissa units are in multiples of $\sigma$, the standard deviation of the corresponding Gaussian noise distribution, indicated by the gray region.

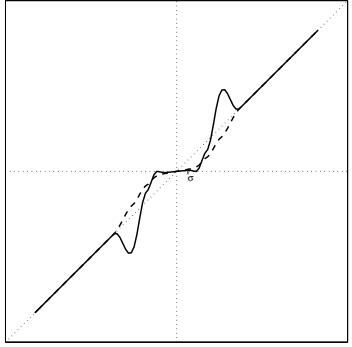

(a)

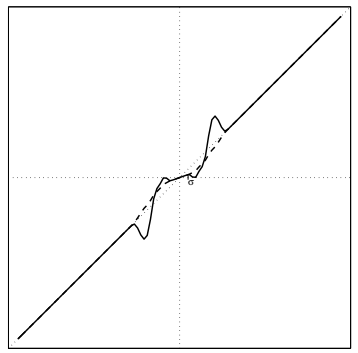

(c)

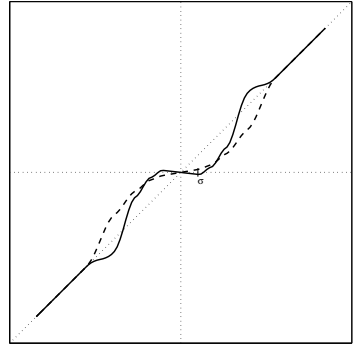

(b)

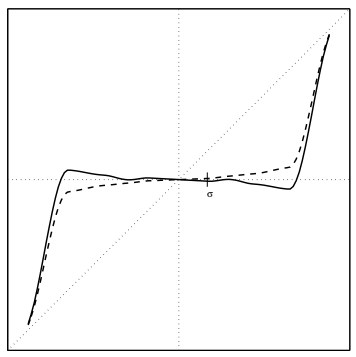

(d)
Fig. 2. Example shrinkage functions, $\hat{x}(y)=y+y \sum g_{\theta}(y)$, for various subbands of an undecimated Haar decomposition, as optimized for SURE in the subband domain (dashed line) and in the image domain (solid line). For midrange bands, (a)-(c), the image-domain estimation functions show more shrinkage of low amplitude coefficients, with a compensatory amplification of midrange values. The high frequency band (d) shows more shrinkage. Note that all functions are equal to the identity for large values, beyond the location of the last bump.

obtain significant improvement in MSE by jointly choosing the $\left\{g_{k}\right\}$ to optimize Eq. (14). However, recall that SURE in either domain is an empirical estimate of the MSE, which, while unbiased, will still suffer from sampling variability that can lead to suboptimal parameter choices. We will use empirical studies to test the effects of this variability on the practical performance of our method.

\section{A. Implementation}

In this section, we examine the empirical behavior of imagedomain and subband-domain SURE denoising. Optimization of the SURE expression in Eq. (14) is greatly simplified if the denoising functions are drawn from a linear family [29], [18], [15], [13]. Specifically, linear parameterization leads to 


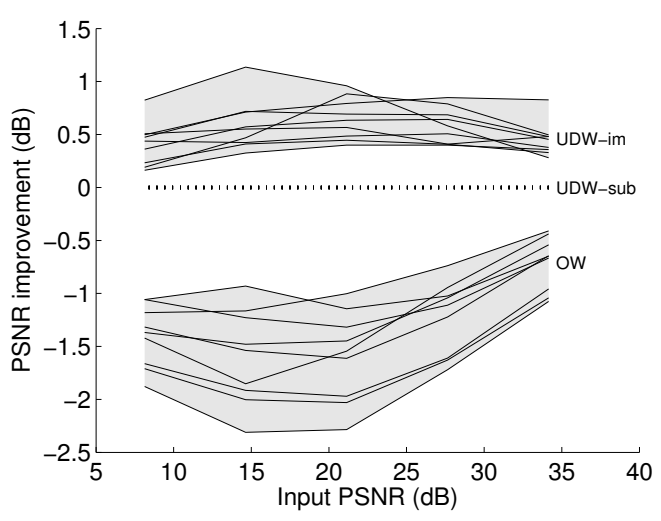

Fig. 3. Comparison of denoising results for scalar SUREbumps denoisers. Each group of lines (indicated by gray regions) shows results for one type of estimator. Each line within a group indicates improvement in PSNR, as a function of input PSNR, for one of nine denoised test images [12]. All results are shown relative to those of SUREbumps applied to undecimated Haar wavelets, optimized in the subband domain (dotted line). Bottom group: SUREbumps applied to orthogonal Haar wavelets; Top group: SUREbumps applied to undecimated Haar wavelets, optimized for image-domain MSE.

a SURE expression that is quadratic in the parameters, and thus easily minimized using standard matrix calculations or gradient-descent methods. We choose a family formed as a linear combination of functions:

$$
g_{k}^{\theta}(y)=y \sum_{m} \theta_{k}^{m} b_{m}(|y| / \sigma)
$$

where

$$
b_{m}(r)=f_{0}(\alpha \cdot \log (r+1)+\beta-m) .
$$

with $f_{0}$ a smooth localized "bump" function

$$
f_{0}(x)= \begin{cases}\cos ^{2}\left(\frac{\pi}{2} x\right), & |x| \leq 1 \\ 0, & |x|>1\end{cases}
$$

$\alpha$, and $\beta$ are chosen to map the centers of the second and last bump to $\sqrt{3} \sigma$ and $\sqrt{15} \sigma$, respectively. The first bump is altered to continue as a constant to the left of the peak (see Fig. 1), providing the denoiser with the capability to eliminate small amplitude coefficients. This family can generate smooth shrinkage functions, including linear estimators, as well as nonlinear "coring" estimators that approximate hard thresholding. In order to restrict the number of parameters that must be optimized, we limit the representation to four bumps, as illustrated in Fig. 1.

To test our methodology, we used decompositions based on a separable Haar basis [30], consisting of local averages and differences of adjacent local averages. These are the simplest (and oldest) of all wavelets, and are easily and efficiently implemented. We used the orthonormal basis, as well as an undecimated decomposition with periodic boundary extension. All images were decomposed into five dyadic scales, which means that the undecimated transform is overcomplete by a factor of 16 .

\section{B. Simulated results}

We computed optimal denoisers by optimizing SURE over the coefficients of the bumps basis, a method which we will

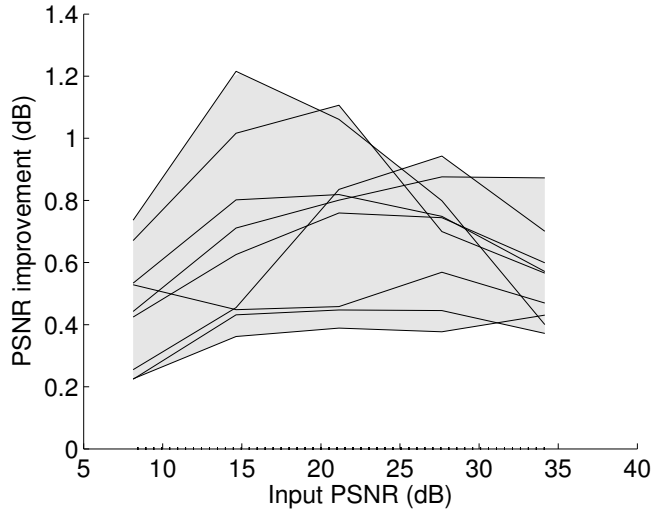

Fig. 4. Performance of SUREbumps optimized in the image domain, relative to SUREshrink (soft thresholding) optimized in the subband domain [2]. Both estimators are applied to coefficients of an undecimated Haar representation. Each line indicates improvement in PSNR (dB) for one of nine test images [12].

refer to as SUREbumps. For the orthonormal case, we optimize Eq. (16), which is equivalent to Eq. (14). For the overcomplete (undecimated) case, we considered estimators optimized in the subband domain using Eq. (16) as well as estimators optimized in the image domain using Eq. (14). Figure 2 shows a comparison of typical shrinkage functions that result from image-domain and subband-domain optimization. Roughly speaking, optimization in the image domain produces stronger suppression of small coefficients, along with preservation (or even boosting) of coefficients of moderate magnitude. That is, we may view the estimator as performing a type of sparsification, as suggested in [7], [21]: lower-amplitude coefficients are suppressed, but medium-amplitude coefficients are boosted in order to compensate for the loss of signal energy. The boosting of mid-amplitude coefficients is also consistent with the findings of [13], in which linearly parameterized marginal shrinkage functions applied to an overcomplete block DCT representation were optimized for image-domain MSE by training on an ensemble of clean images.

Figure 3 shows a comparison of PSNR performance of three methods, across nine test images [12] and a wide range of noise levels. Consistent with the proof of section II, and with empirical results of previous literature, we see that optimizing the denoiser in the subbands of the undecimated basis leads to significant improvement (typically $1.5 \mathrm{~dB}$ ) over the orthonormal basis. Optimizing in the image domain leads to additional improvement (typically $0.5 \mathrm{~dB}$ ).

To give some indication of improvement compared to a well-known result in the literature, we also compared SUREbumps with SUREshrink [31], which is based on soft thresholding functions of the form:

$$
g_{\theta}(y)= \begin{cases}-y, & |y| \leq \theta \\ -\operatorname{sgn}(y) \theta, & |y|>\theta\end{cases}
$$

Although SUREshrink may be optimized for the image domain using Eq. (14), the resulting multi-dimensional objective function is nonconvex, and thus it is not feasible to guarantee global optimality of the solution. Thus, we optimize each threshold parameter $\theta_{k}$ independently by minimizing SURE 


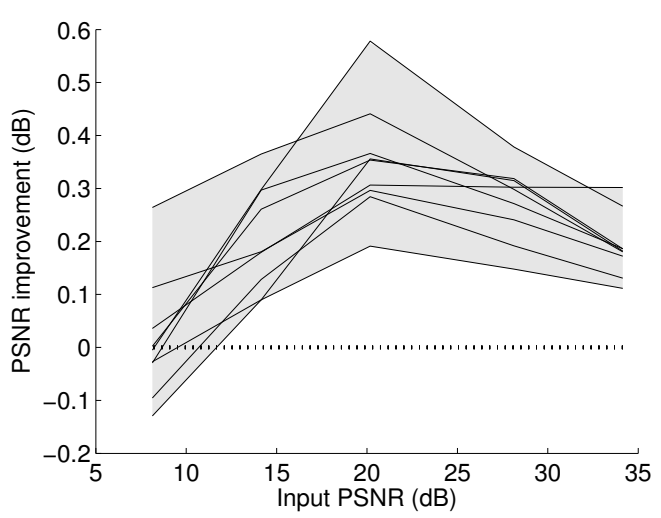

Fig. 5. Performance of SUREbumps estimators on a steerable pyramid decomposition, with 5 scales and 4 oriented subbands at each scale. Lines show performance of SUREbumps optimized in the image domain, relative to SUREbumps optimized in the subband domain.

over subband $\mathcal{S}_{k}$ using Eq. (16), as was done in [2]. Figure 4 shows a comparison across all test images and noise levels, demonstrating that SUREbumps optimized in the image domain offers a substantial improvement over SUREshrink in the transform domain (typically $0.6 \mathrm{~dB}$ ).

\section{Image-domain optimization of other redundant transforms}

Thus far, we have shown examples with orthonormal and undecimated wavelet decompositions, which directly reveal the advantages of introducing redundancy, and of joint optimization in the image domain. However, our method for jointly optimizing the subband denoising functions is valid for any transform with a left inverse, and in particular for all tight frames. As such, we have also examined the behavior of SUREbumps when jointly optimizing estimators applied to a tight frame known as the "steerable pyramid" [23]. The representation is overcomplete by a factor of roughly $4 K / 3+1$, where $K$ is the number of orientation bands utilized. In our tests, we used $K=4$, which produces a transform that is overcomplete by a factor of 6.33 . Figure 5 shows the improvement obtained by using SUREbumps in the image domain compared with SUREbumps in the subband domain. Significant improvement is still obtained, although not quite as much as in the undecimated wavelet case shown in Fig. 3, perhaps because the degree of overcompleteness is much less than for the undecimated wavelet representation. Also notice that at high noise levels (low PSNR), the pyramid-domain method sometimes outperforms the image-domain method. As mentioned earlier, this can arise from sampling errors in SURE, which have more of an effect when trying to jointly optimize the parameters for all the bands, instead of for each band separately.

\section{EXAMPLE: LOCAL CONTEXT ESTIMATORS IN OVERCOMPLETE BASES}

As mentioned in Section II, substantial improvements can be achieved with estimators that operate on neighborhoods of coefficients. We previously defined context estimators in which each coefficient is denoised as a function of its surrounding

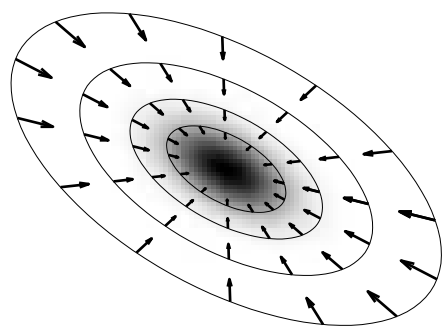

Fig. 6. Illustration of joint shrinkage function, as a vector field in two dimensions, superimposed on a grayscale image representing the underlying Gaussian noise density. Each vector shows the change made to an observed (noisy) vector $\mathbf{u}$. Elliptical contours indicate the maxima of the 4 bump functions, $b_{k}$. The set of vectors $u$ that lie along any one of these contours are denoised by multiplying by a common scalar shrinkage factor. In practice, only the coefficient at the center of each neighborhood is multiplied by this factor.

neighbors, according to Eq. (4). Rewriting this in terms of $g$ gives:

$$
\hat{v}_{i}\left(\mathbf{u}_{i}\right)=u_{i}+g_{k}\left(\mathbf{u}_{i}\right) .
$$

SURE can be extended to handle this situation, by taking into account the fact that coefficients are no longer denoised independently.

To simplify notation and computation, we assume that the neighborhood of each coefficient contains only coefficients from the same subband. Using our previous notation for the inner products of the transform basis functions, we can express the covariance structure of the noise within the subband as

$$
C_{i j}=\sigma^{2} n_{i j} .
$$

Equation (7) implies that to denoise subband $\mathcal{S}_{k}$, we should pick $g_{k}$ to minimize

$$
\sum_{i \in \mathcal{S}_{k}} g_{k}\left(\mathbf{U}_{i}\right)^{2}+2 \sigma^{2} \sum_{i \in \mathcal{S}_{k}} \mathbf{n}_{k} \cdot \nabla g_{k}\left(\mathbf{U}_{i}\right)
$$

where $\mathbf{n}_{k}$ is a vector that contains the inner product of the basis vector corresponding to the coefficient in the center of a neighborhood with every basis function in that neighborhood. Analogous to Eq. (16), the single-band expressions may be additively combined in a single objective function for optimizing the set $\left\{g_{k}\right\}$ in the subband domain:

$$
\sum_{k} \sum_{i \in \mathcal{S}_{k}} g_{k}\left(\mathbf{U}_{i}\right)^{2}+2 \sigma^{2} \sum_{k} \sum_{i \in \mathcal{S}_{k}} \mathbf{n}_{k} \cdot \nabla g_{k}\left(\mathbf{U}_{i}\right)
$$

On the other hand, if we wish to use SURE to denoise in the image domain, Eq. (13) implies we must choose $\left\{g_{k}\right\}$ to minimize

$$
\left|W^{T} g\left(\mathbf{U}_{i}\right)\right|^{2}+2 \sigma^{2} \sum_{k} \sum_{i \in \mathcal{S}_{k}} \mathbf{n}_{k} \cdot \nabla g_{k}\left(\mathbf{U}_{i}\right)
$$

As in the scalar case, the first term takes into account the interactions of the denoising operations when the coefficients are recombined in the image domain.

\section{A. Implementation}

Analogous to the one-dimensional case, we choose a linear family of estimators that operate by shrinking the noisy 


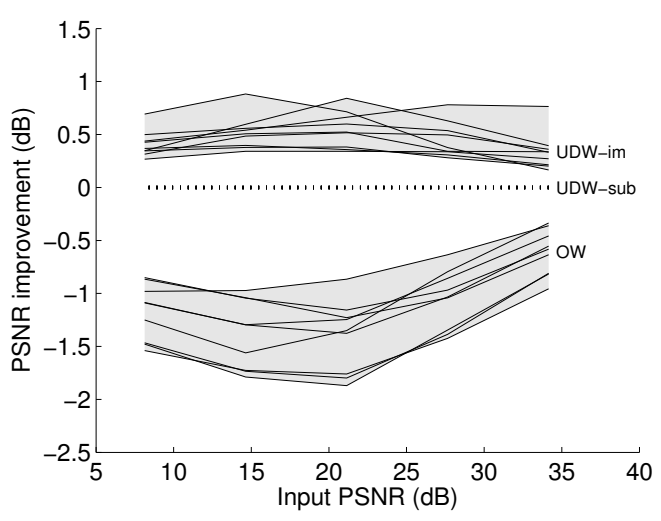

Fig. 7. Comparison of denoising results for vector SUREbumps denoisers. See caption of Fig. 3.

observation by a factor that depends on the amplitude of the observation relative to the noise strength:

$$
\hat{\mathbf{V}}\left(\mathbf{U}_{i}\right)=\mathbf{U}_{i}+\mathbf{U}_{i} \sum_{m} \theta_{m} b_{m}\left(\sqrt{\mathbf{U}_{i}^{T} C_{n}^{-1} \mathbf{U}_{i}}\right)
$$

where $C_{n}$ is the noise covariance in the subband, and the bump functions $b_{k}$ are defined as before by Eq. (18), except that the locations of the first and last bumps are scaled by the square root of the dimension. An example of the type of shrinkage field that results from this parameterization is shown for the two-dimensional case in Fig. 6. As described in Eq. (19) we then use this to denoise the central coefficient:

$$
\hat{V}_{i}\left(\mathbf{U}_{i}\right)=U_{i}+U_{i} \sum_{m} \theta_{m} b_{m}\left(\sqrt{\mathbf{U}_{i}^{T} C_{n}^{-1} \mathbf{U}_{i}}\right)
$$

To illustrate our theorem, we used spatial neighborhoods of size $3 \times 3$ coefficients, located on a subsampled lattice as described in Section II, and boundaries (including neighborhood calculations) are handled with periodic extension.

\section{B. Simulation results}

Figure 7 shows a performance comparison of the vector bumps denoiser optimized individually for subband-domain MSE (using Eq. (21)) and optimized jointly for image-domain MSE (using Eq. (22)). As in the scalar case, and consistent with the proof of Section II, application of SUREbumps in the overcomplete (undecimated) representation leads to a substantial improvement over application in the orthonormal representation (typically $>1 \mathrm{~dB}$ ), and optimization in the image domain offers an additional improvement over optimization in the subband domain (nearly $0.5 \mathrm{~dB}$ ).

\section{Comparison of vector and scalar SUREbumps}

Thus far, we used a neighborhood structure such that the basis vectors associated with the elements of a neighborhood are orthogonal. In this situation, examination of Eq. (22) shows that the MSE estimate is not much different from that of the marginal case, in that it does not make use of the derivative of the denoised coefficient with respect to its neighbors. Consistent with this, we find experimentally that a denoiser based on this neighborhood structure does not show much

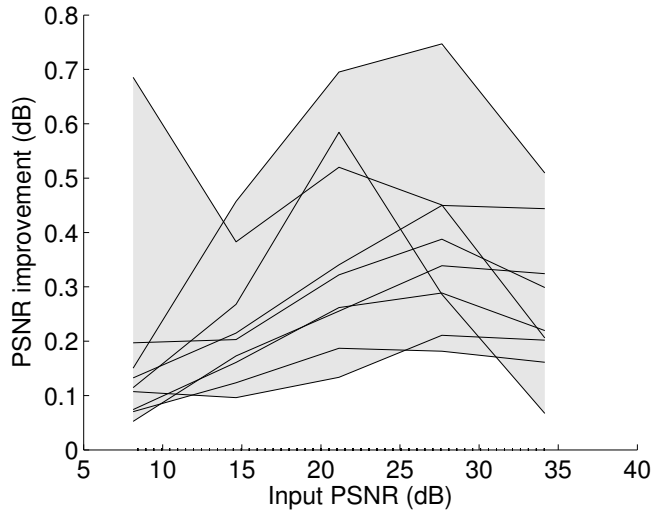

Fig. 8. Comparison of denoising results for vector SUREbumps (solid lines) relative to those for scalar SUREbumps (dotted line). Both estimators are applied to an undecimated Haar decomposition and optimized in the image domain. This vector denoiser uses $3 \times 3$ neighborhoods of adjacent coefficients in the undecimated decomposition.

performance gain compared to a scalar denoiser. For these reasons, we have also implemented a vector denoiser based on neighborhood containing a $3 \times 3$ set of nearest neighbors on the undecimated lattice. Figure 8 shows the improvement of this nearest-neighbor denoiser over the scalar denoiser, which is typically $0.1-0.5 \mathrm{~dB}$.

\section{Comparison to BLS-GSM context denoiser}

To give some indication of improvement relative to another result in the literature, we also applied the BLS-GSM estimator of [12] to the undecimated Haar decomposition using the same neighborhood structure. Note that the originally published BLS-GSM results use a steerable pyramid decomposition, reflected boundary handling, different neighborhood structure near the boundary, and include coarser-scale parents as part of the neighborhood. By enforcing the same decomposition and neighborhood structure, we are providing a direct comparison of the two statistical procedures: BLS-GSM, which is based on an explicit prior model, and SUREbumps, which is prior-free and based on an explicitly parameterized denoising function. Figure 9 shows the improvement in PSNR of using the vector SUREbumps estimator optimized in the image domain, relative to using the BLS-GSM estimator. Despite the simplistic functional form of the estimator used in SUREbumps, performance is typically better than that of BLS-GSM. Table I provides PSNR values of the vector SUREbumps method, optimized in the image domain, for all test images at all noise levels. Although they do not quite achieve the performance level of current state-of-the-art methods (e.g., [28]), the results are roughly comparable to many recent results (e.g., [32], [12], [33], [34]), especially at low to moderate levels of noise. Figure 10 shows example denoised images. Increases in redundancy and image domain optimization are both seen to improve visual quality.

\section{DISCUSSION}

In this article, we have examined the problem of MSEoptimal denoising in overcomplete subband representations. 


\begin{tabular}{|c|c|c|c|c|c|c|c|c|c|}
\hline$\sigma /$ PSNR & Barbara & Boat & Flinstones & House & Lena & Goldhill & Tulips & Parkbench & Mtwill \\
\hline $5 / 34.15$ & 37.13 & 37.21 & 35.93 & 38.84 & 38.38 & 37.08 & 38.58 & 35.98 & 36.53 \\
\hline $10 / 28.13$ & 33.01 & 33.69 & 31.92 & 35.63 & 35.24 & 33.43 & 34.73 & 31.33 & 32.77 \\
\hline $25 / 20.17$ & 27.88 & 29.42 & 27.53 & 31.75 & 31.19 & 29.45 & 30.14 & 26.10 & 28.82 \\
\hline $50 / 14.15$ & 24.41 & 26.34 & 24.02 & 28.15 & 28.18 & 26.85 & 26.75 & 23.05 & 26.13 \\
\hline $100 / 8.13$ & 22.20 & 23.64 & 20.29 & 24.50 & 25.28 & 24.48 & 23.51 & 20.62 & 23.60 \\
\hline
\end{tabular}

TABLE I

DENOISING RESULTS, FOR THE VECTOR SUREBUMPS METHOD, FOR NINE IMAGES AT FIVE DIFFERENT NOISE LEVELS, EXPRESSED AS PEAK-TO-PEAK SIGNAL-TO-NOISE RATIO (PSNR). FOR ALL RESULTS, SUREBUMPS WERE APPLIED TO A FIVE-SCALE UNDECIMATED HAAR DECOMPOSITION, WITH $3 \times 3$ NEIGHBORHOOD OF ADJACENT COEFFICIENTS, OPTIMIZED FOR IMAGE-DOMAIN MSE.

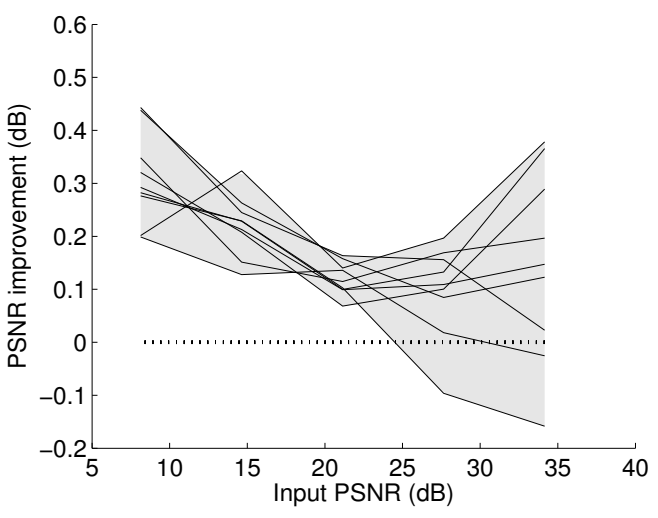

Fig. 9. Performance of vector SUREbumps optimized in the image domain, relative to BLS-GSM estimator [12] (dotted line). Both estimators are applied to coefficients of an undecimated Haar representation. Each line indicates improvement in PSNR (dB) for one of nine test images [12].

We've provided a formal explanation for the empirically observed fact that subband denoising methods can be improved by making the representation more redundant, and we've shown that performance can be further improved by jointly optimizing the subband estimators to minimize imagedomain MSE. In order to examine these effects empirically, we developed generalizations of Stein's unbiased risk estimate to include correlated noise, vector denoisers, and image-domain MSE estimates for denoising functions applied to subbands of overcomplete representations. Using scalar and vector denoisers constructed from linear combinations of "bumps", we have shown through simulations that optimization of imagedomain MSE can lead to substantial performance gains over the suboptimal application of SURE in each subband. These results underscore the importance of distinguishing between the choice of subband decomposition (e.g., orthogonal vs. redundant, separable vs. oriented), the method of denoising (e.g., thresholding vs. BLS-GSM vs. linearly parameterized bumps), the means by which the denoiser parameters are selected (e.g., optimized over a training set vs. maximum likelihood fitting of a prior density vs. SURE) and the domain in which the parameters are optimized (subband vs. image). Comparison of methods that differ in more than one of these factors leaves one unable to conclusively determine the underlying sources of advantage or disadvantage. While it is difficult to formally quantify the interaction of these factors, we believe such understanding would allow for further improvement in performance.

In order to simplify our presentation, we have focused on denoisers operating on rather simple decompositions (Haar wavelets with periodic boundary handling). These decompositions can easily be extended to form overcomplete tight frames by cycle-spinning or eliminating decimation. It is worth investigating the effects of overcompleteness in other bases, particularly oriented redundant bases (e.g., steerable pyramid [23], complex wavelets [25], curvelets [24]). In these cases, it might also prove worthwhile to explore other means of increasing redundancy, such as including rotated or dilated copies of the initial basis set into the transform. If the statistics of subbands are maintained when this redundancy is introduced, the proof of Section II will still guarantee improvement for transform domain denoising. Bases that use reflected boundary handling (as opposed to periodic handling) would also likely lead to improvements in performance. Another issue worth investigating is the choice of neighborhoods on the performance of context denoisers. For example, the inclusion of coefficients from other subbands (e.g., coarser-scale "parents") in the neighborhood has been found to produce substantial increases in performance [11], [12], [15], although a substantial portion of these increases may be obtained through the use of larger spatial neighborhoods [35], [36].

Perhaps more importantly, we believe there is room for substantial improvement in the design of the denoising functions. The examples in this article used a fixed linear family of "bump" functions, whose shapes and positions were crudely hand-optimized to deal with several issues that can affect MSE. First, as in all statistical regression problems, the complexity of the model governs a well-known tradeoff between systematic errors (bias) and generalization error (variance, or overfitting). Specifically, the family of denoising functions should be sufficiently rich to handle a wide variety of source images and noise levels, but it should also be simple enough that its parameters are well constrained by the observed image data. Errors in parameter estimation arise from errors in computing SURE, and these, in turn, depend on both the amount of data (e.g., the number of coefficients in a subband), and the strength of the signal relative to the noise. Second, the choice of denoiser need not rely entirely on the data from the particular image being denoised. Generally, denoising methods include some aspects that are tuned/adapted to the idiosyncrasies of each particular image, and other aspects that are chosen/optimized for performance over image ensembles. In fact, some methods rely entirely on the latter, optimizing over a large set of training images to select a single universal denoiser (e.g., [33], [13]). In our implementation, some prior information is implicitly 
included through the design of the bumps. Prior information could be more explicitly incorporated in the deterministic choice of a function family, and/or through a prior probability model on the parameter values. A principled solution should then trade off between adaptively optimizing to a particular image, whenever the data provide a sufficient constraint, and using the prior information to regularize the solution in cases for which the image data are insufficient.

More generally, the use of SURE for adapting a denoiser to the properties of an observed noisy sample is a particular solution of the "universal" restoration problem, which aims to recover a signal with unknown characteristics from observations corrupted by a process with known characteristics. This problem has been recently explored in several very different forms [37], [27], [38], [39], [29], [28], and it should prove interesting to explore the similarities and relative advantages of these different methods.

\section{REFERENCES}

[1] J. P. Rossi, "Digital techniques for reducing television noise," JSMPTE, vol. 87, pp. 134-140, 1978.

[2] D. Donoho, "Denoising by soft-thresholding," IEEE Trans. Info. Theory, vol. 43, pp. 613-627, 1995.

[3] E. P. Simoncelli and E. H. Adelson, "Noise removal via Bayesian wavelet coring," in Proc 3rd IEEE Int'l Conf on Image Proc, vol. I. Lausanne: IEEE Sig Proc Society, September 16-19 1996, pp. 379-382.

[4] H. A. Chipman, E. D. Kolaczyk, and R. M. McCulloch, "Adaptive Bayesian wavelet shrinkage," J American Statistical Assoc, vol. 92, no. 440, pp. 1413-1421, 1997.

[5] F. Abramovich, T. Sapatinas, and B. W. Silverman, "Wavelet thresholding via a Bayesian approach," J R Stat Soc B, vol. 60, pp. 725-749, 1998.

[6] B. Vidakovic, "Nonlinear wavelet shrinkage with Bayes rules and Bayes factors," Journal of the American Statistical Association, vol. 93, pp. 173-179, 1998.

[7] Hyvarinen, "Sparse code shrinkage: Denoising of nongaussian data by maximum likelihood estimation," Neural Computation, vol. 11, no. 7, pp. $1739-1768,1999$.

[8] M. Figueiredo and R. Nowak, "Wavelet-based image estimation: An empirical Bayes approach using Jeffrey's noninformative prior," IEEE Trans. Image Proc., vol. 10, no. 9, pp. 1322-1331, September 2001.

[9] M. K. Mihçak, I. Kozintsev, K. Ramchandran, and P. Moulin, "Lowcomplexity image denoising based on statistical modeling of wavelet coefficients," Signal Processing Letters, vol. 6, no. 12, pp. 300-303, December 1999.

[10] S. G. Chang, B. Yu, and M. Vetterli, "Spatially adaptive wavelet thresholding with context modeling for image denoising," IEEE Trans. Image Proc., vol. 9, no. 9, pp. 1522-1531, September 2000.

[11] L. Sendur and I. W. Selesnick, "Bivariate shrinkage functions for wavelet-based denoising exploiting interscale dependency," IEEE Trans. Sig. Proc., vol. 50, no. 11, pp. 2744-2756, November 2002.

[12] J. Portilla, V. Strela, M. J. Wainwright, and E. P. Simoncelli, "Image denoising using a scale mixture of Gaussians in the wavelet domain," IEEE Trans Image Processing, vol. 12, no. 11, pp. 1338-1351, November 2003.

[13] Y. Hel-Or and D. Shaked, "A discriminative approach for wavelet shrinkage denoising," IEEE Trans. Image Proc., 2007, to Appear.

[14] C. M. Stein, "Estimation of the mean of a multivariate normal distribution," Annals of Statistics, vol. 9, no. 6, pp. 1135-1151, November 1981.

[15] F. Luisier, T. Blu, and M. Unser, "A new SURE approach to image denoising: Interscale orthonormal wavelet thresholding," IEEE Trans. Image Proc., vol. 16, no. 3, March 2007.

[16] J. C. Pesquet and D. Leporini, "A new wavelet estimator for image denoising," in 6th International Conference on Image Processing and its Applications, Dublin, Ireland, July 1997, pp. 249-253.

[17] A. Benazza-Benyahia and J. C. Pesquet, "Building robust wavelet estimators for multicomponent images using Stein's principle," IEEE Trans. Image Proc., vol. 14, no. 11, pp. 1814-1830, November 2005.
[18] T. Blu and F. Luisier, "The SURE-LET approach to image denoising," IEEE Trans. Image Proc., vol. 16, no. 11, pp. 2778-2786, November 2007.

[19] R. R. Coifman and D. L. Donoho, "Translation-invariant de-noising," in Wavelets and statistics, A. Antoniadis and G. Oppenheim, Eds. San Diego: Springer-Verlag lecture notes, 1995.

[20] E. P. Simoncelli, "Bayesian denoising of visual images in the wavelet domain," in Bayesian Inference in Wavelet Based Models, P. Müller and B. Vidakovic, Eds. New York: Springer-Verlag, 1999, ch. 18, pp. 291308, lecture Notes in Statistics, vol. 141.

[21] M. Elad, "Why simple shrinkage is still relevant for redundant representations?" it, vol. 52, no. 12, Dec 2006.

[22] M. Raphan and E. P. Simoncelli, "Optimal denoising in redundant bases," in Proc 14th IEEE Int'l Conf on Image Proc. San Antonio, TX: IEEE Computer Society, September 2007, best student paper award.

[23] E. P. Simoncelli, W. T. Freeman, E. H. Adelson, and D. J. Heeger, "Shiftable multi-scale transforms," IEEE Trans Information Theory, vol. 38, no. 2, pp. 587-607, March 1992, special Issue on Wavelets.

[24] E. J. Candès and D. L. Donoho, "Curvelets - a surprisingly effective nonadaptive representation for objects with edges," in Curves and Surfaces, C. Rabut, A. Cohen, and L. L. Schumaker, Eds. Nashville, TN: Vanderbilt Univ. Press, 2000, pp. 105- V120.

[25] N. Kingsbury, "Complex wavelets for shift invariant analysis and filtering of signals," Applied and Computational Harmonic Analysis, vol. 10, no. 3, pp. 234-253, May 2001.

[26] G. Fan and X. G. Xia, "Image denoising using a local contextual hidden Markov model in the wavelet domain," IEEE Sig Proc Lett, vol. 8, no. 5, pp. 125-128, May 2001.

[27] A. Buades, B. Coll, and J. M. Morel, "A review of image denoising algorithms, with a new one," Multiscale Modeling and Simulation, vol. 4, no. 2 , pp. 490-530, July 2005.

[28] K. Dabov, A. Foi, V. Katkovnik, and K. Egiazarian, "Image denoising by sparse 3D transform-domain collaborative filtering," IEEE Trans. Image Proc., vol. 16, no. 8, August 2007.

[29] M. Raphan and E. P. Simoncelli, "Learning to be Bayesian without supervision," in Adv. Neural Information Processing Systems 19, B. Schölkopf, J. Platt, and T. Hofmann, Eds., vol. 19. Cambridge, MA: MIT Press, May 2007.

[30] A. Haar, "Zur theorie der orthogonalen funktionensysteme," Math Annal., vol. 69, pp. 331-371, 1910 .

[31] D. Donoho and I. Johnstone, "Adapting to unknown smoothness via wavelet shrinkage," J American Stat Assoc, vol. 90, no. 432, December 1995.

[32] A. Pižurica, W. Philips, I. Lemahieu, and M. Acheroy, "A joint inter- and intrascale statistical model for Bayesian wavelet based image denoising," IEEE Trans. Image Proc., vol. 11, no. 5, pp. 545-557, May 2002.

[33] S. Roth and M. Black, "Fields of experts: A framework for learning image priors," in IEEE Conf. on Computer Vision and Pattern Recognition, vol. II, June 2005, pp. 860-867.

[34] C. Kervrann and J. Boulanger, "Optimal spatial adaptation for patchbased image denoising," IEEE Trans. Image Proc., vol. 15, no. 10, pp. 2866-2878, 2006

[35] J. Liu and P. Moulin, "Information-theoretic analysis of interscale and intrascale dependencies between image wavelet coefficients," IEEE Trans. Image Proc., vol. 10, no. 10, pp. 1647-1658, November 2001.

[36] R. W. Buccigrossi and E. P. Simoncelli, "Image compression via joint statistical characterization in the wavelet domain," IEEE Trans Image Proc, vol. 8, no. 12, pp. 1688-1701, December 1999.

[37] T. Weissman, E. Ordentlich, G. Seroussi, S. Verdú, and M. Weinberger, "Universal discrete denoising: Known channel," IEEE Trans. Info. Theory, vol. 51, no. 1, pp. 5-28, January 2005.

[38] S. P. Awate and R. T. Whitaker, "Unsupervised, information-theoretic, adaptive image filtering for image restoration," IEEE Pat. Anal. Mach. Intell., vol. 28, no. 3, pp. 364-376, March 2006.

[39] M. Elad and M. Aharon, "Image denoising via sparse and redundant representations over learned dictionaries," IEEE Trans. Image Proc., vol. 15, no. 12, pp. 3736-3745, December 2006. 
(a)

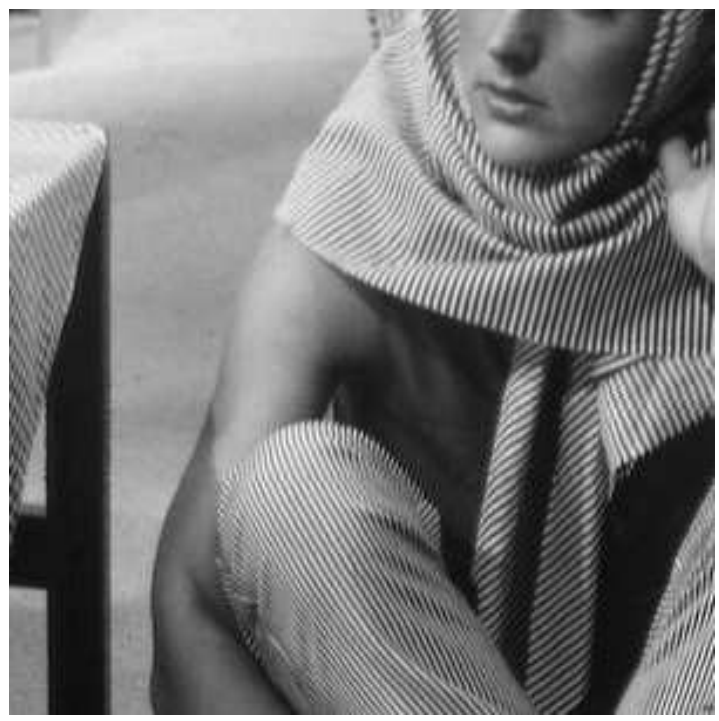

(c)

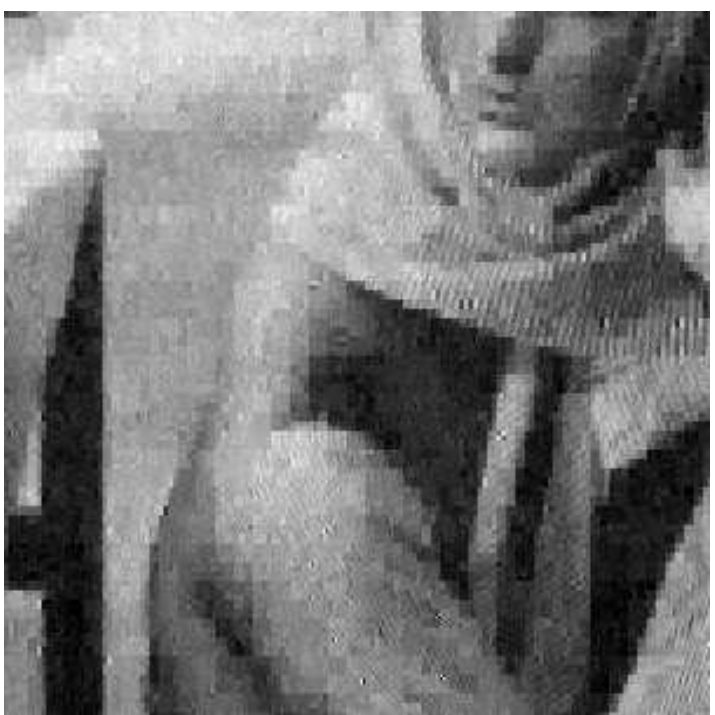

(e)

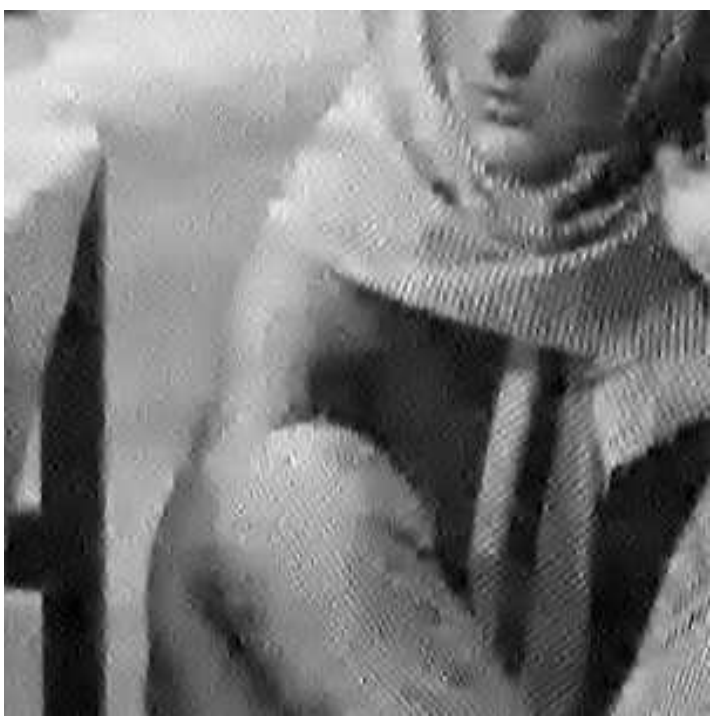

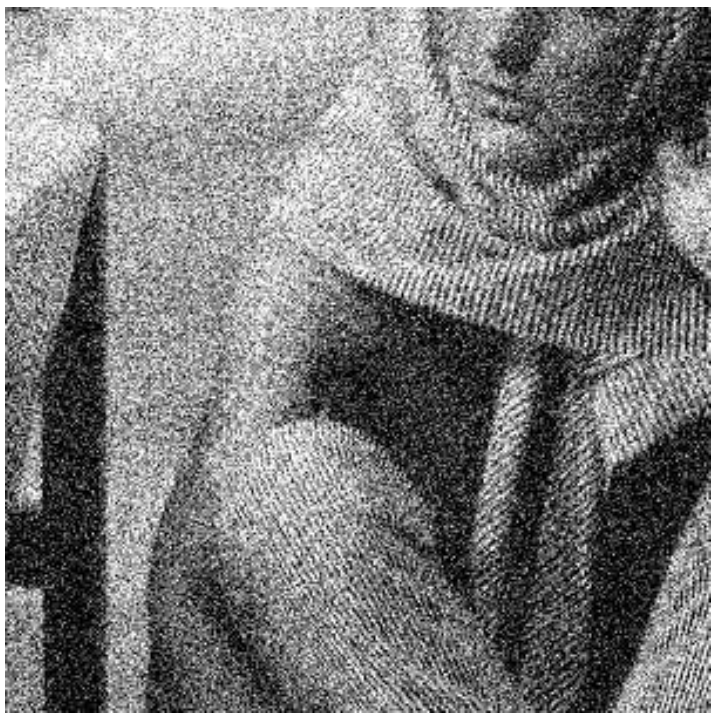

(b)

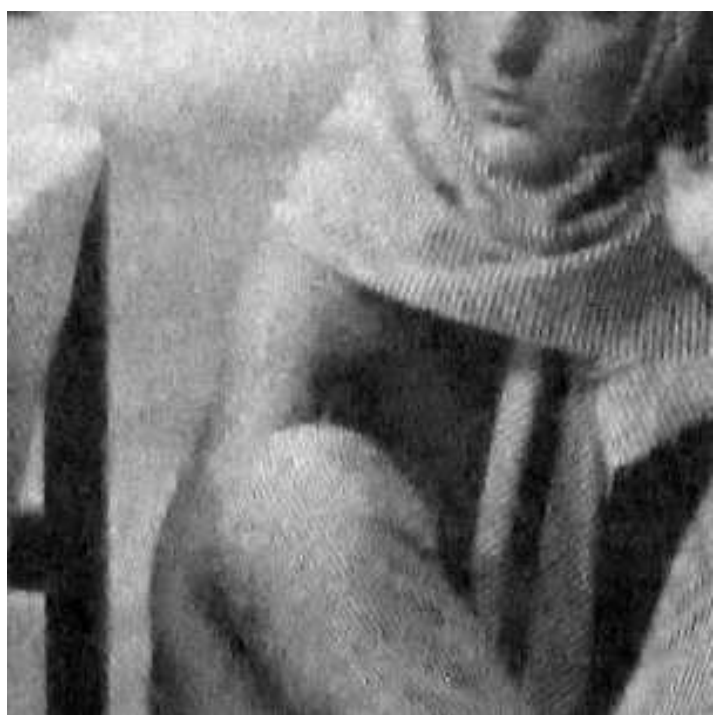

(d)

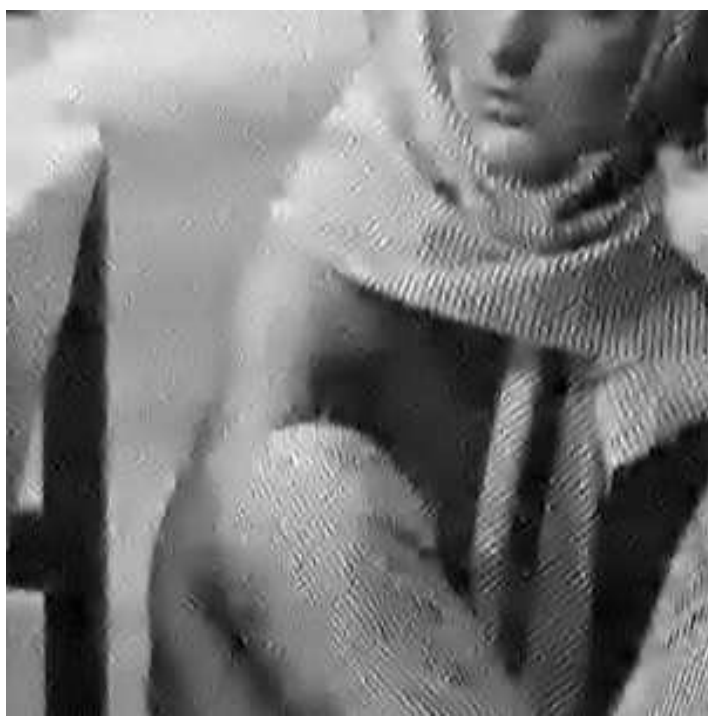

Fig. 10. Denoising results. (a) original image (cropped); (b) noisy ( $15.00 \mathrm{~dB})$; (c) scalar SUREbumps on orthogonal wavelet (22.96 dB); (d) scalar SUREbumps on undecimated wavelet, optimized in the subband domain $(23.89 \mathrm{~dB})$; (e) scalar SUREbumps on undecimated wavelet, optimized in the image domain (24.39 $\mathrm{dB})$; (f) vector SUREbumps on undecimated wavelet, optimized in the image domain (24.86 dB). All methods are applied to a Haar decomposition. 